\title{
Energy Transfer from Quantum Dots to Metal-Organic Frameworks for Enhanced Light Harvesting
}

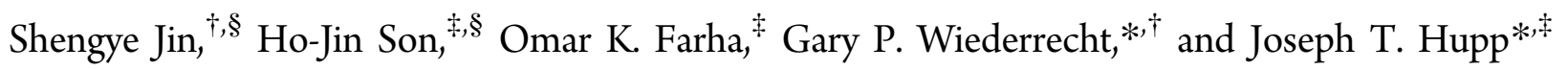

${ }^{\dagger}$ Center for Nanoscale Materials, Argonne National Laboratory, 9700 South Cass Avenue, Argonne, Illinois 60439, United States

${ }^{\ddagger}$ Department of Chemistry, Northwestern University, 2145 Sheridan Road, Evanston, Illinois 60208, United States

\section{Supporting Information}

ABSTRACT: Because of their efficient energy-transport properties, porphyrin-based metal-organic frameworks (MOFs) are attractive compounds for solar photochemistry applications. However, their absorption bands provide limited coverage in the visible spectral range for light-harvesting applications. We report here the functionalization of porphyrin-based MOFs with $\mathrm{CdSe} / \mathrm{ZnS}$ core/ shell quantum dots (QDs) for the enhancement of light harvesting via energy transfer from the QDs to the MOFs. The broad absorption band of the QDs in the visible region offers greater coverage of the solar spectrum by QD-MOF hybrid structures. We show through timeresolved emission studies that photoexcitation of the QDs is followed by energy transfer to the MOFs with efficiencies of more than $80 \%$. This sensitization approach can result in a $>50 \%$ increase in the number of photons harvested by a single monolayer MOF structure with a monolayer of QDs on the surface of the MOF.

$\mathrm{M}$ etal-organic frameworks (MOFs), ${ }^{1}$ which are hybrid materials made from organic linkers and inorganic nodes, have received extensive attention because of their attractive chemical and physical properties, including tunable and extraordinary porosity, ${ }^{2}$ long-distance internal energy migration, ${ }^{3}$ and the capability of catalysis. ${ }^{4}$ These properties lead to numerous potential applications of MOFs in gas storage, ${ }^{5}$ sensing, ${ }^{6}$ chemical separation, ${ }^{7}$ drug delivery, ${ }^{8}$ and so on. In addition to these attractive applications, we are particularly interested in using MOFs as light-harvesting antenna and photosynthesis-like ensembles for solar energy conversion. A few important studies demonstrating the promise of MOFs for conversion of solar energy to electrical or chemical energy have been reported previously. ${ }^{9}$ For example, Lin, Meyer, and co-workers have reported the fabrication and energy-migration dynamics of metal-bipyridine-derived MOFs for light harvesting. ${ }^{10}$ We reported the successful synthesis of metalloporphyrin-derived MOFs for light harvesting and (dark) catalysis. ${ }^{11}$ Since these metalloporphyrin molecules are structurally similar to natural porphyrin-type light-harvesting and photosynthesis pigments, ${ }^{12}$ porphyrin-based MOFs might be expected to mimic important aspects of natural photosynthetic systems. Our previous studies have shown that in a zinc porphyrin-based MOF the photogenerated exciton can migrate over $10-30$ porphyrin struts within its lifetime, with a high anisotropy along a preferred direction. ${ }^{13}$ The long distance and directional exciton migration suggest promising applications of MOFs, superior to monomer dye molecules, as efficient light-harvesting and energy-transport components in solar energy conversion devices.

The efficiency of such a component depends in part on the fraction of photons in the solar spectrum that MOFs can absorb. However, few photoactive molecular building blocks of MOFs are capable of efficiently absorbing photons across the entire solar spectrum. For example, the UV-vis absorption spectra of the zinc porphyrin molecules F-ZnP and DA-ZnP used here to synthesize porphyrin-based MOFs are shown in Figure 1a. Both exhibit Soret $(\lambda=422 \mathrm{~nm}$ for F-ZnP and 448

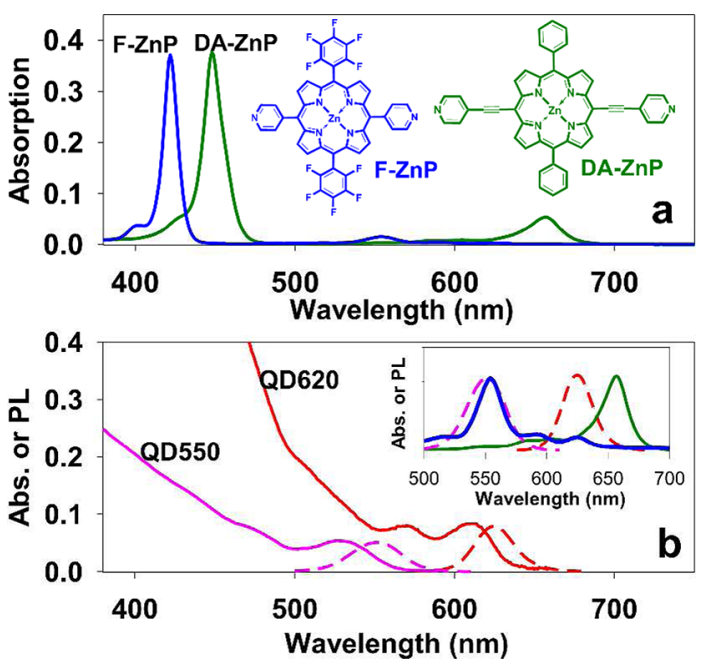

Figure 1. (a) UV-vis absorption spectra (solid lines) of F-ZnP and $\mathrm{DA}-\mathrm{ZnP}$ in toluene and (b) absorption (solid lines) and emission (dashed lines) spectra of CdSe/ZnS core/shell QDs of different sizes (QD550 and QD620) in water. The inset in (a) shows the chemical structures of F-ZnP and DA-ZnP. The inset in (b) compares the normalized emission spectra of the QDs and the Q-band absorptions of $\mathrm{F}-\mathrm{ZnP}$ and DA-ZnP.

$\mathrm{nm}$ for $\mathrm{DA}-\mathrm{ZnP})$ and $\mathrm{Q}(\lambda=555 \mathrm{~nm}$ for $\mathrm{F}-\mathrm{ZnP}$ and $658 \mathrm{~nm}$ for DA-ZnP) absorption bands, but their absorption spectra cover only limited portions of the visible region.

Unlike these molecular building blocks, semiconductor quantum dots (QDs) have broad absorption spectra. They

Received: October 1, 2012

Published: January 7, 2013 
also feature large extinction coefficients, high luminescence quantum yields, and excellent photostabilities. Their optical spectra are systematically tunable via changes in their sizes. ${ }^{14}$ For example, the absorption and emission spectra of two sizes of $\mathrm{CdSe} / \mathrm{ZnS}$ core/shell QDs with emission peaks centered at $550 \mathrm{~nm}$ (named QD550) and $620 \mathrm{~nm}$ (named QD620) are shown in Figure 1b. Compared with the porphyrin molecules, QDs show much broader absorption spectra. These properties make QDs an attractive class of light-harvesting materials. ${ }^{15}$ Huo, Farha, and co-workers have recently reported the incorporation of CdTe QDs within the crystals of a zeolitic imidazolate framework to introduce photoluminescence (PL) properties in MOFs. ${ }^{16}$ However, the incorporation of QDs with MOFs to enhance light harvesting has not been explored.

In this communication, we report a strategy of using semiconductor QDs to enhance light harvesting by MOFs through energy transfer from the QDs to the MOFs. The strategy is illustrated in Figure 2. The $\mathrm{CdSe} / \mathrm{ZnS}$ core/shell

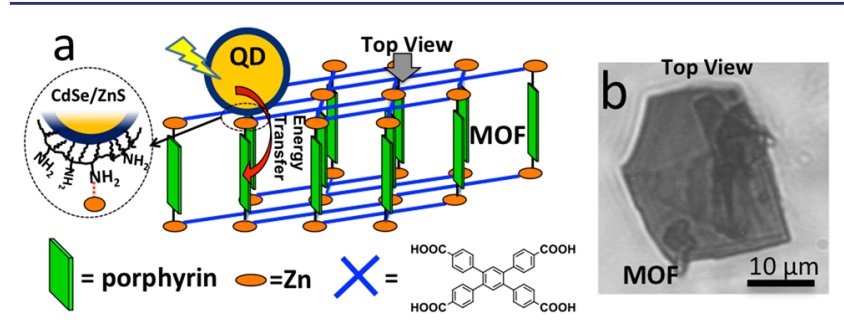

Figure 2. (a) Schematic diagram of a QD-MOF complex and (b) optical microscopy image of a plate-shaped MOF particle. The surface plane of the MOF contains the 1,2,4,5-tetrakis(4-carboxyphenyl)benzene moieties, and $\mathrm{Zn}$ metal centers connect the porphyrin blocks. The number of porphyrin molecular layers determines the thickness of the MOF particle. The CdSe/ZnS QDs are sensitized on the MOF surface through the amine- $\mathrm{Zn}$ interaction. The QD-MOF hybrid is able to harvest photons through energy transfer from the QDs to the MOF even in spectral regions where the MOF has little absorption.

QDs are sensitized on the surface of the porphyrin-based MOFs through the amine functional groups. The QDs are used to harvest photons in the spectral region where the MOFs have little absorption. The photon-generated excitons in the QDs are then transferred to the MOFs through resonance energy transfer. Such a QD-MOF hybrid can harvest photons well beyond the absorption spectrum of the MOF. In this work, the effectiveness of such a strategy was examined via time-resolved fluorescence measurements, by which we found that the time required for energy transfer from the QDs to the MOFs can be $1-2$ ns with quantum efficiencies of $>80 \%$. Furthermore, the observation of PL of MOFs due to the energy transfer from QDs confirmed the light harvesting by the QD-MOF hybrids even in the spectral regions where the MOFs have little absorption.

The porphyrin molecules $\mathrm{F}-\mathrm{ZnP}$ and $\mathrm{DA}-\mathrm{ZnP}$ (Figure 1a inset) and their corresponding MOFs (named F-MOF and DAMOF, respectively) were synthesized according to the methods described in the Supporting Information (SI). The powder Xray diffraction (PXRD) patterns of the MOFs and space-filling models of the structures are shown in Figures $\mathrm{S} 1$ and $\mathrm{S} 2$ in the SI, respectively. The QD550 and QD620 CdSe/ZnS core/shell QDs were purchased from Ocean Nanotech. These QDs were coated by a monolayer of amphiphilic polymer with amine functional groups and a monolayer of poly(ethylene glycol) (PEG). ${ }^{17}$ The spectral overlaps between the emissions of the
QDs and the Q-band absorptions of the MOFs (Figure $1 \mathrm{~b}$ inset) indicate that resonant energy transfer from the QDs (donor) to the MOFs (acceptor) is possible. To sensitize the QDs on the external surfaces of the MOFs, the MOF samples were first dried in an oven at $70^{\circ} \mathrm{C}$ for $1 \mathrm{~h}$ and then exposed to aqueous $\mathrm{QD}$ solutions $(0.8 \mu \mathrm{M})$ for $1 \mathrm{~h}$. The $\mathrm{QD}$-sensitized MOFs were finally dropped and dried on the surface of glass coverslips. Typical TEM images of DA-MOFs before and after QD sensitization (Figure S3) show the coating of QDs on the surface of the QD-MOF hybrids. It is believed that the binding of the QDs on the MOFs is due to amine- $\mathrm{Zn}$ coordination (see Figure 2). The fluorescence lifetimes and emission spectra of the QD-MOF complexes were obtained using a home-built confocal microscope system coupled with a time-correlated single-photon counting module, with $\lambda_{\mathrm{ex}}=400 \mathrm{~nm}$ (the frequency-doubled output of our Ti:sapphire laser) at a power of $30 \mathrm{nW}$. Details of the experimental setup are provided in the SI and shown in Figure S4.

The strategy of QD-enhanced light harvesting by MOFs was first examined by time-resolved fluorescence measurements. The fluorescence decay curves of QD550- and QD620sensitized MOFs are shown in Figure 3. For measurements,
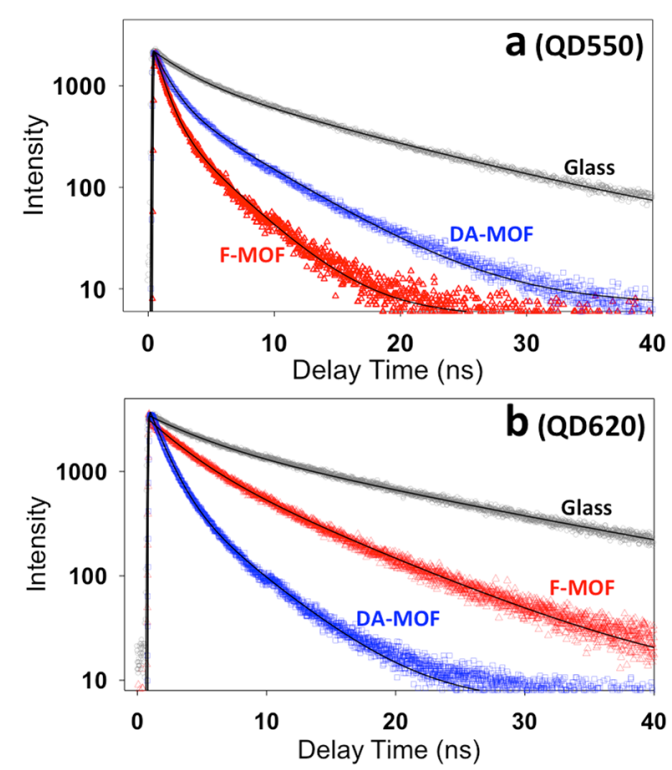

Figure 3. Fluorescence decays of (a) QD550 and (b) QD620 on various substrates (F-MOF, DA-MOF, and glass). Black solid lines are fits of the decays according to eq $\mathrm{S} 1$.

specific optical filters before the detector were used to ensure that the decay curves were constructed from only the photons emitted from the QDs (see the SI and Figures S5 and S6 for details). The lifetimes of both QD samples on both MOFs were dramatically shorter than on glass. The shortened lifetimes of the QDs could in principle be due to either charge transfer or energy transfer between the QDs and the MOFs. Electron transfer from the QDs to the MOFs is not energetically allowed, as shown in the energy diagram in Figure S7. Charge transfer from the MOFs to the QDs is also ruled out because the fluorescence lifetimes of the MOFs were not changed by the presence of the QDs. The possibility of energy transfer from the QDs to free porphyrin molecules that may dissolve from the MOFs was also excluded by our control experiments (see Figure S8). We therefore attribute the shortened lifetimes of the QDs to energy transfer from the QDs to the MOFs. 
The assignment to energy transfer is supported by the spectral overlaps between the emissions of the QDs and the Qband absorptions of the MOFs (Figure 1b). For QD550 (Figure 3a), the lifetime of the QDs on F-MOF is shorter than on DA-MOF, indicating faster energy-transfer dynamics on FMOF. This observation is consistent with the relatively large spectral overlap between QD550 and F-MOF. In contrast to QD550, QD620 offers a larger spectral overlap with DA-MOF, hence leading to a shorter lifetime (faster energy transfer) on DA-MOF than on F-MOF. Additionally, in the fluorescence decay curves of DA-MOFs (Figure S9), the MOF excited through energy transfer from QDs exhibits a much longer kinetic rise time than the MOF excited directly by the laser, indicating the generation of excitons. This confirms the energy transfer from QDs to MOFs.

To resolve the energy-transfer dynamics quantitatively, the fluorescence decays in Figure 3 were fit with a biexponential function (eq S1 in the SI), and the lifetimes of QDs on different substrates were calculated according to eq S2. The average lifetimes of QD550 and QD620 on glass were 8.0 and $11.4 \mathrm{ns,}$ respectively. The fluorescence lifetimes of the QDs on glass and MOF are $\tau_{\text {glass }}=1 /\left(k_{\mathrm{r}}+k_{\mathrm{nr}}\right)=1 / k_{0}$ and $\tau_{\mathrm{MOF}}=1 /\left(k_{0}+k_{\text {eng }}\right)$, respectively, in which $k_{\mathrm{r}}$ and $k_{\mathrm{nr}}$ are the radiative and nonradiative decay constants, respectively, and $k_{\text {eng }}$ is the energy-transfer rate constant. The quantum yield of energy transfer is then given by $\phi_{\text {eng }}=k_{\text {eng }} /\left(k_{0}+k_{\text {eng }}\right)$. By comparison of the lifetimes of QDs on glass and the MOFs, the energytransfer times $\left(=1 / k_{\text {eng }}\right)$ and $\phi_{\text {eng }}$ values for the QDs on the two MOFs were calculated (Table 1). The energy-transfer times

Table 1. Average Lifetimes, Energy-Transfer Times, and Quantum Yields of QD550 and QD620 Sensitized on FMOF and DA-MOF

\begin{tabular}{ccccccccc} 
& \multicolumn{3}{c}{ F-MOF } & & \multicolumn{3}{c}{ DA-MOF } \\
\cline { 2 - 4 } & $\tau_{\text {ave }}(\mathrm{ns})$ & $1 / k_{\text {eng }}(\mathrm{ns})$ & $\phi_{\text {eng }}$ & & $\tau_{\text {ave }}(\mathrm{ns})$ & $1 / k_{\text {eng }}(\mathrm{ns})$ & $\phi_{\text {eng }}$ \\
QD550 & 1.4 & 1.7 & $82 \%$ & & 2.7 & 4.1 & $66 \%$ \\
QD620 & 5.2 & 9.6 & $54 \%$ & & 1.8 & 2.1 & $84 \%$ \\
\hline
\end{tabular}

from QD550 to F-MOF and DA-MOF were 1.7 and $4.1 \mathrm{~ns}$, respectively, and those for QD620 were 9.6 and $2.1 \mathrm{~ns}$, respectively. The $\phi_{\text {eng }}$ values were as high as $82 \%$ for QD550 on F-MOF and 84\% for QD620 on DA-MOF. Thus, efficient energy transfer from the QDs to the MOFs clearly is possible.

We hypothesized that the sensitization of QDs on MOFs is spatially facilitated by coordination of available $\mathrm{Zn}$ metal centers on the external surfaces of the MOFs by the amine functional groups on the surfaces of the QDs (Figure 2). To confirm this interaction between the QDs and MOFs, the fluorescence decays of similar $\mathrm{CdSe} / \mathrm{ZnS}$ QDs without surface amine functional groups were also measured on F-MOF. These QDs have an emission spectrum and luminescence quantum yield very similar to those of QD550. The samples were prepared using the same procedures as mentioned above, and the QDs were believed to be physically attached to the MOF surface. The energy-transfer time for these QDs on F-MOF was calculated to be $8.0 \mathrm{~ns}$ (Figure S10), which is much greater than the time of $1.7 \mathrm{~ns}$ for QD550 on the same MOF. This comparison suggests that the amine functional groups may help the QDs to be closely attached on the MOF surface through amine-Zn interactions, leading to a higher energy-transfer rate.

To examine further the light harvesting by MOFs through energy transfer from QDs, the PL spectra of the MOFs and
QD-MOF complexes were measured (Figure 4). At $\lambda_{\text {ex }}=400$ $\mathrm{nm}$, PL for F-MOF was clearly observed (Figure 4a-1), while

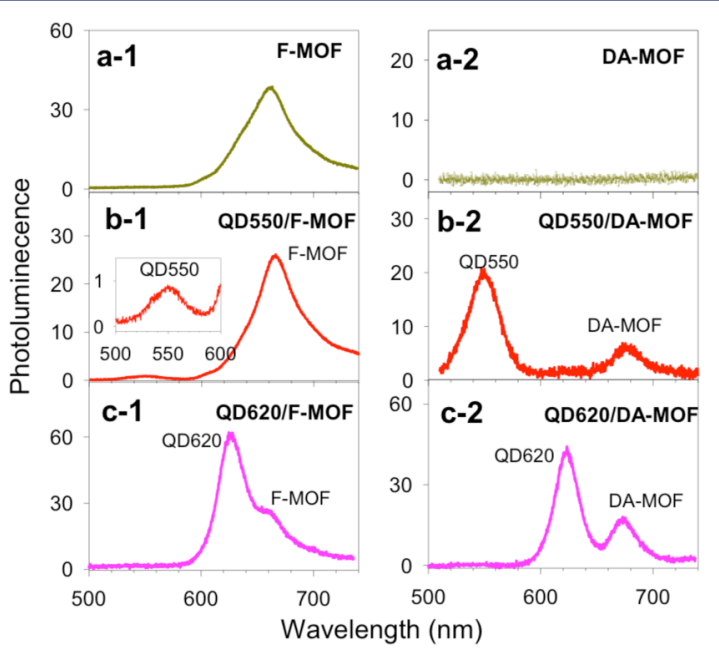

Figure 4. Emission spectra of the MOFs and QD-MOF complexes at $\lambda_{\mathrm{ex}}=400 \mathrm{~nm}$ : (a-1) F-MOF; (a-2) DA-MOF; (b-1) QD550-F-MOF; (b-2) QD550-DA-MOF; (c-1) QD620-F-MOF; (c-2) QD620-DAMOF. The inset in (b-1) is an expanded view of the QD550 emission.

no PL signal was detected from DA-MOF (Figure 4a-2). These observations are consistent with the absorption spectra of F$\mathrm{ZnP}$ and DA-ZnP (Figure 1a), which show that DA- $\mathrm{ZnP}$ is hardly excited at $400 \mathrm{~nm}$. For the QD-F-MOF complexes, the emissions from both the QDs and F-MOF were detected (Figure 4b-1 for QD550 and Figure 4c-1 for QD620). The emissions of QDs correspond to the remaining fluorescence signals after quenching by energy transfer to the MOF. Since FMOF itself has PL at $\lambda_{\mathrm{ex}}=400 \mathrm{~nm}$, the PL from F-MOF due to energy transfer is not resolvable in Figure $4 b-1, c-1$. In the PL spectra of the QD-DA-MOF complexes (Figure $4 \mathrm{~b}-2$ for QD550 and Figure 4c-2 for QD620), the DA-MOF emission peak centered at $680 \mathrm{~nm}$ was observed in addition to the peaks from the QDs. Since DA-MOF itself has no detectable PL signal at $\lambda_{\text {ex }}=400 \mathrm{~nm}$ (Figure 4a-2), the PL from DA-MOF in the QD-DA-MOF complexes must be due to energy transfer from the QDs. This confirms the light harvesting by the QDMOF hybrids through energy transfer from the QDs to the MOF even at wavelengths where the MOF has little or no absorption. In view of the low PL quantum yield of DA-MOF (0.096), the DA-MOF emission peaks shown in Figure 4b-2,c-2 correspond to the harvesting of $\sim 2.2 \times 10^{5}$ and $\sim 4.6 \times 10^{5}$ photons/s by the QD550-DA-MOF and QD620-DA-MOF hybrids, respectively, under our experimental conditions.

Taking the QD620-DA-MOF complex with the highest $\phi_{\text {eng }}$ as an example, the enhancement in MOF light harvesting can be quantitatively estimated through calculations (see the SI for the details). For a DA-MOF presenting a single external monolayer of porphyrin building blocks, a monolayer of QD620 enables the MOF to harvest $~ 51 \%$ more photons under one-sun conditions. Considering that the exciton can migrate at least 10 net porphyrin struts in DA-MOF along preferred directions (this value limits the thickness of the MOF to ensure efficient energy delivery in practical applications), ${ }^{13}$ a more realistic enhancement capacity of $5-10 \%$ is estimated for DA-MOFs with a thickness of 10 porphyrin layers. One would expect more efficient energy transfer from QDs to DA-MOF and hence a greater enhancement of light harvesting if the QD 
emission could be more red-shifted to improve the spectral overlap. Such tuning could be achieved simply by changing the size of the QDs. QD550 and QD620 were particularly selected in this study for the convenience of detection in the separation of the PL from the QDs and that from the MOFs.

In summary, we report here a QD-based strategy for enhancing light harvesting of appropriately designed MOFs. With CdSe/ZnS core/shell QDs chemically bound to their exterior surfaces, F-MOF and DA-MOF are able to function as Förster-type energy acceptors, allowing the QD-MOF hybrids to harvest photons even at wavelengths where the MOFs have little or no absorption. Energy transfer with quantum efficiencies of up to $84 \%$ was achieved by tuning the size of the QDs, thereby increasing the spectral overlap between the QD emission and MOF absorption. This work offers a pathway for utilizing QDs to compensate for the relatively narrow absorption spectra of the molecular building blocks of singlechromophore MOFs and paves the road for the design and development of QD-MOF complexes that efficiently harvest light for solar energy conversion.

\section{ASSOCIATED CONTENT}

\section{S Supporting Information}

Synthesis of MOFs, detailed description of the experimental setup and fluorescence lifetime measurements, fitting parameters of lifetime decay curves, diagram of energy levels, and additional fluorescence decay curves of QDs. This material is available free of charge via the Internet at http://pubs.acs.org.

\section{AUTHOR INFORMATION}

\section{Corresponding Author}

wiederrecht@anl.gov; j-hupp@northwestern.edu

\section{Author Contributions}

${ }^{\S}$ S.J. and H.-J.S. contributed equally.

\section{Notes}

The authors declare no competing financial interest.

\section{ACKNOWLEDGMENTS}

J.T.H. gratefully acknowledges support from the U.S. Department of Energy, Office of Science, Office of Basic Energy Sciences (DOE-BES) under Grant DE-FG02-87ER13808 (work involving the synthesis of molecular materials). S.J. acknowledges support from the ANSER Center, an Energy Frontier Research Center funded by DOE-BES under Award DE-SC0001059 (work involving QDs and/or time-resolved fluorescence spectroscopy). Use of the Center for Nanoscale Materials and support of G.P.W. was funded by DOE-BES through Contract DE-AC02- 06CH11357.

\section{REFERENCES}

(1) (a) Férey, G. Chem. Soc. Rev. 2008, 37, 191. (b) Tranchemontagne, D. J.; Mendoza-Cortes, J. L.; O'Keeffe, M.; Yaghi, O. M. Chem. Soc. Rev. 2009, 38, 1257.

(2) (a) Bradshaw, D.; Warren, J. E.; Rosseinsky, M. J. Science 2007, 315, 977. (b) Kitagawa, S.; Kitaura, R.; Noro, S. Angew. Chem., Int. Ed. 2004, 43, 2334. (c) Evans, O. R.; Lin, W. B. Acc. Chem. Res. 2002, 35, 511. (d) Ockwig, N. W.; Delgado-Friedrichs, O.; O’Keeffe, M.; Yaghi, O. M. Acc. Chem. Res. 2005, 38, 176.

(3) Kent, C. A.; Liu, D.; Meyer, T. J.; Lin, W. J. Am. Chem. Soc. 2012, 134, 3991.

(4) (a) Lee, J.; Farha, O. K.; Roberts, J.; Scheidt, K. A.; Nguyen, S. T.; Hupp, J. T. Chem. Soc. Rev. 2009, 38, 1450. (b) Ma, L.; Abney, C.; Lin,
W. Chem. Soc. Rev. 2009, 38, 1248. (c) Ma, L.; Falkowski, J. M.; Abney, C.; Lin, W. Nat. Chem. 2010, 2, 838.

(5) (a) Farha, O. K.; Yazaydin, A. O.; Eryazici, I.; Malliakas, C. D.; Hauser, B. G.; Kanatzidis, M. G.; Nguyen, S. T.; Snurr, R. Q.; Hupp, J. T. Nat. Chem. 2010, 2, 944. (b) Eddaoudi, M.; Kim, J.; Rosi, N.; Vodak, D.; Wachter, J.; O’Keeffe, M.; Yaghi, O. M. Science 2002, 295, 469. (c) Furukawa, H.; Miller, M. A.; Yaghi, O. M. J. Mater. Chem. 2007, 17, 3197. (d) Kaye, S. S.; Dailly, A.; Yaghi, O. M.; Long, J. R. J. Am. Chem. Soc. 2007, 129, 14176. (e) Lin, X.; Telepeni, I.; Blake, A. J.; Dailly, A.; Brown, C. M.; Simmons, J. M.; Zoppi, M.; Walker, G. S.; Thomas, K. M.; Mays, T. J.; Hubberstey, P.; Champness, N. R.; Schroeder, M. J. Am. Chem. Soc. 2009, 131, 2159. (f) Murray, L. J.; Dincă, M.; Long, J. R. Chem. Soc. Rev. 2009, 38, 1294. (g) Rosi, N. L.; Eckert, J.; Eddaoudi, M.; Vodak, D. T.; Kim, J.; O’Keeffe, M.; Yaghi, O. M. Science 2003, 300, 1127. (h) Sava, D. F.; Kravtsov, V. C.; Eckert, J.; Eubank, J. F.; Nouar, F.; Eddaoudi, M. J. Am. Chem. Soc. 2009, 131, 10394.

(6) (a) Xie, Z. G.; Ma, L. Q.; deKrafft, K. E.; Jin, A.; Lin, W. B. J. Am. Chem. Soc. 2010, 132, 922. (b) Allendorf, M. D.; Houk, R. J. T.; Andruszkiewicz, L.; Talin, A. A.; Pikarsky, J.; Choudhury, A.; Gall, K. A.; Hesketh, P. J. J. Am. Chem. Soc. 2008, 130, 14404. (c) Chen, B.; Wang, L.; Xiao, Y.; Fronczek, F. R.; Xue, M.; Cui, Y.; Qian, G. Angew. Chem., Int. Ed. 2009, 48, 500. (d) Lan, A.; Li, K.; Wu, H.; Olson, D. H.; Emge, T. J.; Ki, W.; Hong, M.; Li, J. Angew. Chem., Int. Ed. 2009, 48, 2334.

(7) Li, J.-R.; Kuppler, R. J.; Zhou, H.-C. Chem. Soc. Rev. 2009, 38, 1477.

(8) (a) An, J.; Geib, S. J.; Rosi, N. L. J. Am. Chem. Soc. 2009, 131, 8376. (b) Horcajada, P.; Serre, C.; Vallet-Regi, M.; Sebban, M.; Taulelle, F.; Férey, G. Angew. Chem., Int. Ed. 2006, 45, 5974.

(9) (a) Kobuke, Y. Eur. J. Inorg. Chem. 2006, 2333. (b) Lee, C. Y.; Farha, O. K.; Hong, B. J.; Sarjeant, A. A.; Nguyen, S. T.; Hupp, J. T. J. Am. Chem. Soc. 2011, 133, 15858.

(10) Kent, C. A.; Liu, D.; Ma, L.; Papanikolas, J. M.; Meyer, T. J.; Lin, W. J. Am. Chem. Soc. 2011, 133, 12940.

(11) (a) Farha, O. K.; Shultz, A. M.; Sarjeant, A. A.; Nguyen, S. T.; Hupp, J. T. J. Am. Chem. Soc. 2011, 133, 5652. (b) Shultz, A. M.; Farha, O. K.; Hupp, J. T.; Nguyen, S. T. J. Am. Chem. Soc. 2009, 131, 4204.

(12) (a) Barber, J.; Andersson, B. Nature 1994, 370, 31. (b) McDermott, G.; Prince, S. M.; Freer, A. A.; HawthornthwaiteLawless, A. M.; Papiz, M. Z.; Cogdell, R. J.; Isaacs, N. W. Nature 1995, 374, 517. (c) Kuhlbrandt, W.; Wang, D. N.; Fujiyoshi, Y. Nature 1994, 367,614 .

(13) Son, H.-J.; Jin, S.; Patwardhan, S.; Wezenberg, S. J.; Jeong, N. C.; So, M.; Wilmer, C. E.; Sarjeant, A. A.; Schatz, G. C.; Snurr, R. Q.; Farha, O. K.; Wiederrecht, G. P.; Hupp, J. T. J. Am. Chem. Soc. 2012, DOI: $10.1021 /$ ja310596a.

(14) Kongkanand, A.; Tvrdy, K.; Takechi, K.; Kuno, M.; Kamat, P. V. J. Am. Chem. Soc. 2008, 130, 4007.

(15) (a) Kamat, P. V. J. Phys. Chem. C 2008, 112, 18737. (b) Hodes, G. J. Phys. Chem. C 2008, 112, 17778.

(16) Lu, G.; Li, S.; Guo, Z.; Farha, O. K.; Hauser, B. G.; Qi, X.; Wang, Y.; Wang, X.; Han, S.; Liu, X.; DuChene, J. S.; Zhang, H.; Zhang, Q.; Chen, X.; Ma, J.; Loo, S. C. J.; Wei, W. D.; Yang, Y.; Hupp, J. T.; Huo, F. Nat. Chem. 2012, 4, 310.

(17) Yang, L. L.; Mao, H.; Wang, Y. A.; Cao, Z. H.; Peng, X. H.; Wang, X. X.; Duan, H. W.; Ni, C. C.; Yuan, Q. G.; Adams, G.; Smith, M. Q.; Wood, W. C.; Gao, X. H.; Nie, S. M. Small 2009, 5, 235. 